\title{
Open Cosmologies with Rotation
}

\author{
Saulo Carneiro 1
}

Received May 11, 2001

\begin{abstract}
We study a rotating and expanding, Gödel type metric, originally considered by Korotkii and Obukhov [1,2], showing that, in the limit of large times and nearby distances, it reduces to the open metric of Friedmann. In the epochs when radiation or dust matter dominate the energy density, our solutions are similar to the isotropic ones and, in what concerns processes occurring at small times, the rotation leads only to higher order corrections. At large times, the solution is dominated by a decaying positive cosmological term, with negative pressure, and necessarily describes a quasi-flat universe if the energy conditions have to be satisfied. The absence of closed time-like curves requires a superior limit for the global angular velocity, which appears as a natural explanation for the observed smallness of the present rotation. The conclusion is that the introduction of a global rotation, in addition to be compatible with observation, can enrich the standard model of the Universe, explaining issues like the origin of galaxies rotation and the quasi-flatness problem.
\end{abstract}

KEY WORDS: Universe model; rotation; angular momentum; closed timelike curves.

\section{INTRODUCTION}

Around ten years ago, Korotkii and Obukhov presented a class of rotating and expanding, Gödel type cosmological metrics [1,2], showing that they respect the observed isotropy of the cosmic background radiation and do not lead to parallax effects. Furthermore, for some values of the metric parameters, there are no closed time-like curves and, then, these metrics do not suffer the causal problems characteristic of the original Gödel's metric [3].

\footnotetext{
${ }^{1}$ Permanent address: Instituto de Física, Universidade Federal da Bahia, 40210-340, Salvador, Bahia, Brazil.saulo@fis.ufba.br
} 
In this paper we will show that, due to conservation of angular momentum, the metric of Korotkii and Obukhov leads, in the limit of large times, to an anisotropic metric that reduces to the open metric of Friedmann in the nearby approximation. For small times, we present an approximate solution valid in the limit of small rotation, which presents an isotropic distribution of pressures and the same evolution law as in the corresponding isotropic case. Due to the rotation, the expressions for the energy density and pressure are affected only by higher order corrections relative to the standard, isotropic expressions, which guarantees that anisotropy does not affect, unless by higher order corrections, the processes occurred during early times.

For the epoch dominated by dust matter, the corresponding expanding solution of Einstein equations is similar to the isotropic open solution, except for an anisotropic distribution of pressures that, as we shall see, can be related to a material content formed by an imperfect fluid. Nevertheless, the anisotropy gives rise to an important difference in the later stages of Universe evolution. For our solutions to satisfy the dominant energy conditions, namely, positivity and causal flux of energy, the epoch dominated by dust matter should naturally be followed by an era of coasting evolution, in which the energy density $\epsilon$ falls with $a^{2}$, where $a$ is the radius of the Universe. This corresponds to a material content that satisfies the equation of state $p=-\epsilon / 3$. Such a content can be interpreted as a decaying positive cosmological term, and it is very significant that arguments from quantum cosmology also predict the conservation law $\epsilon a^{2}=$ constant for a time dependent cosmological term [4]. Moreover, during this phase, the relative energy density (the energy density relative to the critical one) is a constant, and the energy conditions impose a lower bound on its value which is close to the present value. This constitutes a possible explanation for the observed quasi-flatness of the Universe.

The general conclusion we will try to establish is that the introduction of a global rotation into the Universe description, in addition to agree with the observations that have been sustaining the standard model, can shed light on subjects like the origin of galaxies rotation, as pointed out by Li [5], or the quasi-flatness problem.

\section{GÖDEL TYPE METRICS}

The Gödel type metric that we will consider is given by $[1,2,6]$

$$
d s^{2}=a^{2}(\eta)\left[\left(d \eta+l e^{x} d y\right)^{2}-\left(d x^{2}+e^{2 x} d y^{2}+d z^{2}\right)\right],
$$

where $a$ is a scale factor, $l$ is a positive parameter, $\eta$ is the conformal time and $x$, $y, z$ are spatial coordinates.

Korotkii and Obukhov [1,2] have shown that this metric respects the observed isotropy of the cosmic background radiation and does not lead to parallax effects, 
contrary to what would be expected from an anisotropic, rotating metric. Moreover, they have shown that, for $l<1$, there are no causal problems, because the closed time-like curves characteristic of Gödel's metric can appear only for $l>1$ (the Gödel metric corresponds to $l=\sqrt{2}$, with $a$ constant $^{2}$ ).

Metric (1) describes an expanding and rotating universe, with an angular velocity given, in comoving coordinates, by $\omega=l / 2 a[1,2]$. Although this result was derived by Korotkii and Obukhov for a constant value of $l$, it is easy to verify that it remains valid when $l$ is a function of time. Using conservation of angular momentum, it is possible to see that, in the radiation dominated epoch, the parameter $l$ is a constant, as originally considered by Korotkii and Obukhov, while in the matter dominated one it falls with $a$. Indeed, from the conservation of angular momentum, we have $\epsilon \omega a^{5}=$ constant, where $\epsilon$ is the energy density of the matter content. In the radiation epoch, $\epsilon$ falls with $a^{4}$, and so $\omega$ falls with $a$, leading to a constant $l$. On the other hand, for the matter epoch $\epsilon$ falls with $a^{3}$, so $\omega$ falls with $a^{2}$, and $l$ should fall with $a$. As we shall see, in a rotating and expanding universe described by metric (1), the matter dominated epoch should be followed by an era in which the energy density falls with $a^{2}$, if the energy conditions have to be satisfied. So, during this last epoch $\omega$ falls with $a^{3}$, and $l$ falls with $a^{2}$.

Therefore, for large times the terms in $l$ can be dismissed in Einstein's equations, which means to consider, instead of metric (1), the anisotropic metric

$$
d s^{2}=a^{2}(\eta)\left[d \eta^{2}-\left(d x^{2}+e^{2 x} d y^{2}+d z^{2}\right)\right] .
$$

The cosmological solutions we will present in this paper, approximate solutions of metric (1), are exact solutions of the diagonal metric (2), in the particular case $l=0$.

With help of the coordinate transformation

$$
\begin{gathered}
e^{x}=\cosh \xi+\cos \phi \sinh \xi, \\
y e^{x}=\sin \phi \sinh \xi,
\end{gathered}
$$

metric (2) can also be written as

$$
d s^{2}=a^{2}(\eta)\left(d \eta^{2}-d \xi^{2}-\sinh ^{2} \xi d \phi^{2}-d z^{2}\right) .
$$

The coordinate transformation (3) is a particular case, for $l=0$, of a more general transformation, with help of which the metric (1) can be expressed in cylindrical coordinates $[3,7,8]$.

It is easy to show that, in the limit of nearby distances, that is, up to subdominant terms in sinh $\xi$, metric (4) reduces to the open FLRW metric. Indeed, using the transformations

\footnotetext{
${ }^{2}$ For an exhaustive study of the stationary case of metric (1), see the pioneer work of Rebouças and Tiomno [7].
} 


$$
\begin{gathered}
\sinh \xi=\sinh \chi \sin \theta, \\
z=\sinh \chi \cos \theta,
\end{gathered}
$$

relating cylindrical and spherical coordinates, we obtain by differentiation

$$
\begin{gathered}
\cosh \xi d \xi=\sinh \chi \cos \theta d \theta+\cosh \chi \sin \theta d \chi, \\
d z=-\sinh \chi \sin \theta d \theta+\cosh \chi \cos \theta d \chi
\end{gathered}
$$

So, by using

$$
\frac{1}{\cosh ^{2} \xi}=\frac{1}{1+\sinh ^{2} \xi} \approx 1-\sinh ^{2} \xi=1-\sinh ^{2} \chi \sin ^{2} \theta,
$$

we have

$$
d z^{2}+d \xi^{2} \approx \sinh ^{2} \chi d \theta^{2}+d \chi^{2} .
$$

Finally, substituting (8) and the first of equations (5) into (4) leads to

$$
d s^{2} \approx a^{2}(\eta)\left[d \eta^{2}-d \chi^{2}-\sinh ^{2} \chi\left(d \theta^{2}+\sin ^{2} \theta d \phi^{2}\right)\right],
$$

which is precisely the open FLRW metric in spherical coordinates [9].

The requirement of absence of closed time-like curves can be used to understand why the present observed superior limit for the global angular velocity is so small. As said before, the rotation parameter $l$ is given by $l=2 \omega a[1,2]$. Then, the causality condition $l<1$ applied to the radiation dominated era implies that $\omega_{d}<1 / 2 a_{d} \approx 2.5 \times 10^{-15} s^{-1}$, where $\omega_{d}$ is the global angular velocity at the time of decoupling between matter and radiation, and $a_{d}$ is the radius of the Universe at that time, $a_{d} \approx 6 \times 10^{22} \mathrm{~m}$ [10] (for a present radius of the Universe given by $a \approx 1 / H \sim 10^{26} \mathrm{~m}$, where $H$ is the Hubble parameter). As we have seen, during the matter dominated era $\omega a^{2}=$ constant, which leads to an upper limit for the present angular velocity of matter given by $\omega=\omega_{d} a_{d}^{2} / a^{2} \sim 10^{-21} s^{-1}$, while for radiation we obtain, from $\omega_{\mathrm{rad}} a=$ constant, the upper limit $\omega_{\text {rad }}=\omega_{d} a_{d} / a \approx 1.5 \times 10^{-18} s^{-1}$. In this way, the absence of closed time-like curves appears as a natural explanation for the smallness of the present rotation.

\section{THE RADIATION DOMINATED ERA}

From metric (1) and considering $l$ as a function of time, we obtain the Einstein equations

$$
\epsilon a^{4}=-\left(1-\frac{3 l^{2}}{4}\right) a^{2}+3\left(1-l^{2}\right) \dot{a}^{2}-2 l i a \dot{a},
$$




$$
\begin{gathered}
p_{1} a^{4}=\left(\frac{l^{2}}{4}+\dot{l}^{2}+l \ddot{l}\right) a^{2}+\left(1-l^{2}\right) \dot{a}^{2}-2\left(1-l^{2}\right) a \ddot{a}+4 l i a \dot{a}, \\
p_{2} a^{4}=\frac{l^{2}}{4} a^{2}+\left(1-l^{2}\right) \dot{a}^{2}-2\left(1-l^{2}\right) a \ddot{a}+2 l i a \dot{a}, \\
p_{3} a^{4}=\left(1-\frac{l^{2}}{4}+\dot{l}^{2}+l \ddot{l}\right) a^{2}+\left(1-l^{2}\right) \dot{a}^{2}-2\left(1-l^{2}\right) a \ddot{a}+4 l i \dot{a} \dot{a} .
\end{gathered}
$$

Here, $\epsilon$ is the energy density, and $p_{i}, i=1,2,3$, are the principal pressures. The dot means derivation with respect to the conformal time. The other non-null components of the energy-momentum tensor are proportional to $l$, which from now on will be considered a small parameter, i.e., $l<<1$. In this approximation, these non-diagonal components can be neglected compared to the diagonal ones.

As suggested in section 2, let us adopt for the radiation dominated era the ansatz $l=$ constant. The above equations turn out to be

$$
\begin{gathered}
\epsilon a^{4}=-\left(1-\frac{3 l^{2}}{4}\right) a^{2}+3\left(1-l^{2}\right) \dot{a}^{2}, \\
p_{1} a^{4}=p_{2} a^{4}=\frac{l^{2}}{4} a^{2}+\left(1-l^{2}\right) \dot{a}^{2}-2\left(1-l^{2}\right) a \ddot{a}, \\
p_{3} a^{2}=p_{1} a^{2}+1-\frac{l^{2}}{2} .
\end{gathered}
$$

Substituting in (14) the conservation law for radiation, $\epsilon a^{4}=a_{0}^{2}=$ constant, and considering the limit $a \rightarrow 0$, we obtain the solution

$$
a=b \eta=\sqrt{2 b t},
$$

where $t$ is the cosmological time, defined by $d t=a d \eta$, and

$$
b=\frac{a_{0}}{\left[3\left(1-l^{2}\right)\right]^{1 / 2}} \text {. }
$$

This is the same evolution law obtained by the isotropic model in the limit of small times [9].

For the energy density, we then have

$$
\epsilon=\frac{a_{0}^{2}}{a^{4}}=\frac{3}{4 t^{2}}\left(1-l^{2}\right),
$$

while it follows, from equations (15) and (16), in the same limit $a \rightarrow 0$,

$$
p_{i}=p=\frac{\epsilon}{3},
$$


$i=1,2,3$, i.e., the equation of state for radiation, as expected.

For $l<1$, equations (19) and (20) give $\epsilon>0$ and $\left|p_{i}\right|<\epsilon$, that is, the energy conditions [11] are satisfied. For $l^{2}<<1$, those equations give the same predictions as the isotropic model [9]. Another remarkable point is that, although the distribution of pressures is in general anisotropic, in the limit of small times we obtain an isotropic pressure. In addition, the Hubble parameter at this epoch has the same time dependence as in the standard model, namely $H=1 / 2 t$, which leads to the same ratio between the interaction rate and the expansion one. The conclusion is that the thermal history of this universe is the same predicted by the standard model. In what concerns processes occurring during the initial stages of Universe evolution, the anisotropy (and the rotation) may be manifest only as higher order corrections.

\section{COSMOLOGICAL SOLUTIONS FOR LARGE TIMES}

As pressure and energy density decrease, the radiation dominated era evolves until matter and radiation decouple from each other and one enters a matter dominated epoch characterized by the conservation law

$$
\epsilon a^{3}=2 a_{1},
$$

where $a_{1}$ is a constant.

Adopting the ansatz $l a=$ constant, taking the limit of large $a$ and keeping only the dominant terms, the Einstein equations (10)-(13) reduce to

$$
\begin{gathered}
\epsilon a^{3}=-a+\frac{3 \dot{a}^{2}}{a}, \\
p_{1} a^{3}=p_{2} a^{3}=\frac{\dot{a}^{2}}{a}-2 \ddot{a}, \\
p_{3} a^{2}=p_{1} a^{2}+1 .
\end{gathered}
$$

Substituting (21) into (22), we have the solution

$$
a(\eta)=a_{1}\left[\cosh \left(\frac{\eta}{\sqrt{3}}\right)-1\right],
$$

where we have absorbed an integration constant by a suitable shift in the origin of conformal time $\eta$.

With this solution, the spatial Einstein equations (23)-(24) lead to the pressures

$$
\begin{gathered}
p_{1} a^{2}=p_{2} a^{2}=-\frac{1}{3}, \\
p_{3} a^{2}=\frac{2}{3},
\end{gathered}
$$


whose average yields the equation of state for dust matter, $p=0$, as expected.

Hence, the evolution of this universe since the initial, radiation dominated epoch until the matter dominated one is similar to that predicted by the open isotropic model, except for an anisotropy in the pressure distribution, anisotropy that is negligible at early times and that, for large times, is as small as the pressures themselves.

However, there is an important difference with respect to the isotropic case. In the matter dominated era, the energy density falls as $a^{-3}$, while the pressures decrease as $a^{-2}$. So, for large times, the magnitude of the pressures would become larger than the energy density and, consequently, the dominant energy conditions $\epsilon \geq\left|p_{i}\right|$ would not be fulfilled. It is possible to prove that, for the energy conditions to be satisfied at present, the relative energy density should be larger than or equal to 0.4 , but, even so, these conditions would be violated sooner or later in the future.

Therefore, in this anisotropic scenario, the dust era should be followed by an epoch in which the energy density falls, at least, so slowly as $a^{-2}$, that is, according to the conservation law

$$
\epsilon a^{2}=3 b^{2}-1=\text { constant, }
$$

where $b$ is a positive constant introduced for mathematical convenience (the possibility that $b$ be negative would correspond to a contracting universe and will not be studied here).

Substituting this conservation law into Eq. (10) and dismissing the terms in $l$ gives $\dot{a} / a=b$, which leads to the solution

$$
a=e^{b \eta}=b t .
$$

The Hubble parameter is now given by $H=b / a$ and, for the relative energy density, we obtain the constant value

$$
\Omega \equiv \frac{\epsilon}{3 H^{2}}=\frac{3 b^{2}-1}{3 b^{2}} .
$$

The spatial Einstein equations give

$$
\begin{gathered}
p_{1} a^{2}=p_{2} a^{2}=-b^{2}, \\
p_{3} a^{2}=1-b^{2} .
\end{gathered}
$$

For the average pressure, we then get $p=-\epsilon / 3$, an equation of state corresponding to a (decaying) positive cosmological term. In this sense, it is interesting to note that, for a cosmological term varying with time, the conservation law $\epsilon a^{2}=$ constant has also been suggested on the basis of quantum cosmology considerations [4].

It is easy to see that the dominant energy conditions $\epsilon \geq\left|p_{i}\right|$ are now fulfilled provided that $b^{2} \geq 1 / 2$. From (29), one can see that this corresponds to the 
condition $\Omega \geq 1 / 3 \approx 0.3$. On the other hand, it is possible to check that, during the radiation and matter dominated epochs, the relative energy density decreases monotonically, which means that the bound obtained above is a lower limit for $\Omega$ at all times. So, the energy conditions impose a lower bound on the relative energy density, maintaining the Universe in a quasi-flat configuration.

The conservation law $\epsilon a^{2}=$ constant is also compatible with Einstein equations in the isotropic case. For the open FLRW metric, instead of Eq. (10), we have the Friedmann equation [9]

$$
\epsilon=\frac{3}{a^{4}}\left(\dot{a}^{2}-a^{2}\right) \text {. }
$$

Substituting $\epsilon a^{2}=3\left(b^{2}-1\right)$, it is easy to arrive once more at the evolution law (28). In addition, the spatial Einstein equations give $p=-\epsilon / 3$, corresponding again to a (decaying) positive cosmological term. In this case, however, although the energy conditions are satisfied only if $b \geq 1$, no positive lower bound is imposed on the relative energy density, contrary to what happens in the anisotropic case.

More generally, it is possible to prove that the conservation law demanding that $\epsilon a^{2}$ be a constant leads to Eq. (28) for all (open, flat and closed) metrics, in both isotropic and anisotropic cases (actually, in the flat case the anisotropic metric reduces to the isotropic one). Remarkably, however, it is only in the open anisotropic case that the energy conditions impose a quasi-flat configuration.

Let us also note that the anisotropic model presented in this paper does not exclude the possibility of an inflationary phase in the cosmic evolution. Indeed, if we add a dominant, positive cosmological constant to the left hand side of Eq. (10), we obtain an exponential evolution law for $a(t)$. Actually, the introduction of a typical cosmological constant (or, alternatively, the introduction of an energy density falling more slowly than $a^{-2}$ ) in Einstein equations would be needed if recent claims about the observation of a positive cosmic acceleration were confirmed $[12,13]$. As we have shown, a cosmological term decaying as $a^{-2}$ leads to the solution (28), for which the deceleration parameter is exactly zero.

\section{THE MATTER CONTENT AS AN IMPERFECT FLUID}

As we have seen in section 3 , in the limit of small times the matter content of space-time (1) consists of rotating relativistic matter, with energy density and isotropic pressure given by (19) and (20), respectively. For large times $l \rightarrow 0$, and from (11)-(13) we have

$$
p_{1} a^{2}=p_{2} a^{2}=p_{3} a^{2}-1 \text {. }
$$

In this way, we have an anisotropic distribution of pressures, with the anisotropy corrections falling as $a^{-2}$. It is this fact that ultimately leads to the 
necessity of considering a coasting evolution (i.e., $a \propto t$ ) in the last phase of Universe history, if we want to respect the energy conditions in this rotating and expanding context.

The appearance of an anisotropic distribution of pressures and, in particular, of tensions, shows that the material content of our rotating and expanding universe cannot be a perfect fluid, as usual in the standard isotropic cosmologies. Therefore, it is necessary to find a suitable matter source compatible with such an anisotropy in order to put this non-stationary, rotating cosmology on an acceptable physical basis.

Without closing the door to other possibilities [14], a natural candidate for the material content is an homogeneous imperfect fluid with viscosity. Actually, in general situations this choice is more realistic than a perfect fluid. The use of a perfect fluid in standard cosmology is possible due to the isotropy of the fluid motion, which avoids the appearance of friction. But in anisotropic contexts like the one considered here the role of viscosity cannot, in general, be neglected.

In order to simplify our analysis, let us consider the fluid motion in a locally comoving Lorentz frame, that is, a freely falling frame whose origin is at rest with respect to a given point of the fluid at a given time $t$ (owing to the presence of friction, we cannot introduce a globally comoving frame). The energy-momentum tensor of the fluid is given by $[15,16]$

$$
T^{\mu \nu}=-p g^{\mu \nu}+(p+\epsilon) U^{\mu} U^{\nu}+\Delta T^{\mu \nu},
$$

where $p$ is the average pressure and, in our frame, $\Delta T^{00}=0$ and

$$
\begin{gathered}
\Delta T^{i 0}=-\chi_{0}\left(\partial^{i} T+T \dot{U}^{i}\right), \\
\Delta T^{i j}=-\eta_{0}\left(\partial^{j} U^{i}+\partial^{i} U^{j}-\frac{2}{3} \partial_{k} U^{k} \delta^{i j}\right) .
\end{gathered}
$$

Here, $U^{\mu}$ is the fluid velocity, $T$ its temperature, the overdot means derivation with respect to $t$ and $\partial^{i}=\delta^{i j} \partial_{j} . \chi_{0}$ and $\eta_{0}$ are the fluid heat conductivity and shear viscosity, respectively.

In our model with cylindrical symmetry, the second of equations (34) leads to

$$
\begin{gathered}
\Delta T^{11}=\Delta T^{22}=-\frac{2}{3} \eta_{0} h, \\
\Delta T^{33}=\frac{4}{3} \eta_{0} h,
\end{gathered}
$$

where we have defined $h \equiv \partial^{1} U^{1}-\partial^{3} U^{3}=\partial^{2} U^{2}-\partial^{3} U^{3}$. So, we have

$$
\Delta T^{11}=\Delta T^{22}=\Delta T^{33}-2 \eta_{0} h .
$$

Comparing this equation with (32), we see that viscosity gives the expected contribution to the pressures provided that 


$$
2 \eta_{0} h a^{2}=1
$$

Since the anisotropic metric (2) is diagonal, the energy-momentum tensor will also be diagonal, that is, $T^{i 0}=\Delta T^{i 0}=0$. This can be achieved if we consider a null heat conductivity, or an approximately null fluid temperature, or yet if the condition $\partial^{i} T=-T \dot{U}^{i}$ follows. In this last case, since for the coasting solution considered in the last section we have $\dot{U}^{i}=0$, we get $\partial_{i} T=0$, which means isotropy and homogeneity of fluid temperature.

On the other hand, we have $\Delta T^{i j}=0$ for $i \neq j$, which leads to $\partial^{j} U^{i}=$ $-\partial^{i} U^{j}$ for $i \neq j$. This condition can be satisfied, in particular, by the Hubble type law $U_{i}=H_{i} x_{i}, i=1,2,3$. In this case, equation (37) shows that $H_{3}<H_{1}=H_{2}$, and it is important to verify how much this inequality can affect the observed Hubble law.

The relation between the Hubble parameter $H$ and the parameters $H_{i}$ can be established with the help of the equation [16] $\dot{V} / V=\partial_{i} U^{i}$, relating the temporal variation of a volume $V$ of the fluid and the divergence of its velocity field. Considering $V$ as the volume of the observed universe, proportional to $a^{3}$, this leads to $\partial_{i} U^{i}=3 \dot{a} / a=3 H$. So, from $U_{i}=H_{i} x_{i}$, we obtain $H=$ $\left(H_{1}+H_{2}+H_{3}\right) / 3$, that is, the Hubble parameter is equal to the average of the parameters $H_{i}$.

During the coasting phase of Universe evolution, the Hubble parameter is given by $H=b / a$ and, therefore, its relative variation with direction is given by $h / H=\left(2 \eta_{0} b a\right)^{-1}$. If we consider a constant viscosity $\eta_{0}$ (or at least a viscosity varying very slowly with $a$ ), the relative variation of $H$ falls with $a$ and could be unobservable for large times, even for a small value of $\eta_{0}$.

Therefore, the above analysis shows that the solutions found for the spacetime metrics (1) and (2) may correspond to a physical matter content formed by radiation, viscous matter and a cosmological term. The appearance of anisotropic pressures, far from discard the model and render it unphysical, can be related to the presence of friction owing to the anisotropic motion of matter.

\section{A SINGULARITY-FREE ALTERNATIVE SCENARIO}

In a recent paper [17], we have investigated an alternative, singularity free, scenario for Universe's evolution, in which the present expanding universe is originated from a primordial Gödel universe [3], by a phase transition during which the negative cosmological term characteristic of the Gödel phase crosses a positive maximum and rolls down to zero. This scenario could also explain the origin of galaxies rotation, but it was not clear how the global angular momentum of the Universe could be transferred to the galaxies [18]. This difficult is intimately connected to the discontinuous transition considered in that paper, where the Gödel metric (1) (with $l=\sqrt{2}$ ) is directly matched with the expanding (but non-rotating) anisotropic metric (2). 
The analysis we have made in the present paper can help us solving these difficulties. Initially we have a Gödel universe, which corresponds to $l=\sqrt{2}$ and $\epsilon a^{2}=1$. After the phase transition, we have a rotating and expanding universe, in which $l$ (and then the non-diagonal term in metric (1)) falls down, leading to the diagonal metric (2). During the phase transition, half of the initial energy is used to compensate the negative cosmological constant present in the Gödel model, given by $\lambda a^{2}=-1 / 2$. After the phase transition, we then have $\epsilon a^{2}=1 / 2$, relation which allows us to match the original Gödel universe with our last solution (27)-(28) for $b^{2}=1 / 2$, that is, for $\Omega \approx 0.3$. In this way, the scale factor $a$ changes continuously in the whole evolution, and the dominant energy conditions are satisfied. Moreover, the decaying, positive cosmological term characteristic of the expanding phase can be shown to arise naturally from the scalar field transition described in [17], in which a self-interaction potential, initially at a negative minimum (corresponding to the negative cosmological constant present in the Gödel solution), crosses a positive maximum and rolls down to zero. Now, we can use the mechanism proposed by Li to transfer angular momentum from Universe to galaxies in a rotating and expanding context [5].

In the above match, the value of the radius of the primordial universe is not fixed by the present values of the energy density and Hubble parameter, contrary to what occurs in the match considered in Ref. [17] (there, the Gödel phase is matched with the dust solution given by (21) and (25)). In the Gödel phase we have the angular velocity $\omega_{G}=\sqrt{2} / 2 a_{G}$, where $a_{G}$ is the radius of the primordial Gödel's universe. In the expanding phase, the angular velocity of matter at the present time is given by $\omega a^{2}=\omega_{G} a_{G}^{2}$. Substituting this last equation into the former, we obtain $a_{G}=\sqrt{2} \omega a^{2}$, which determines $a_{G}$ for a given value of the present angular velocity of matter.

As already discussed in [17], in this context there is no dense phase, which constitutes the major drawback of this alternative scenario, if we have into consideration phenomena like nucleosynthesis or the cosmic background radiation. Although such phenomena could be related to the very process of phase transition [17], this possibility needs to be further investigated.

\section{CONCLUDING REMARKS}

In this paper we have tried to show that the inclusion of rotation into the standard model of the Universe can enrich it in several aspects. On one hand, rotation does not contradict the current observations of isotropy nor gives rise to parallax effects $[1,2]$; the anisotropic metric (2), that we have just shown to originate from the rotating metric (1) by conservation of angular momentum, reduces to the open metric of Friedman in the limit of nearby distances; in the limit of small times the distribution of pressures is isotropic, and the smallness of the 
rotation parameter $l$ guarantees that physical processes taking place at early times are not affected by rotation, as well as the absence of closed time-like curves.

On the other hand, the global rotation can be used to explain the origin of galaxies rotation and the observed relation between their angular momenta and masses [5]; in the anisotropic context described by metrics (1) and (2), the energy conditions lead naturally to a last epoch dominated by a positive cosmological term decaying as $a^{-2}$, a decaying law also expected on the basis of quantum cosmology reasonings [4]; finally, in such a context, the energy conditions impose also a constant lower bound on the relative energy density, close to the present observed value, providing in this way a possible explanation for the observed quasi-flatness. By the way, let us note that these two last results are originated from the anisotropy of metric (2), no matter its relation with the rotating metric (1).

As recently pointed out [19], the coasting evolution law $a=b t$, characteristic of the last phase of the present model, can solve other cosmological problems as well. For example, it leads to $t=1 / H$, an age for the Universe compatible with the observational bounds. In addition, the conservation law $\epsilon a^{2}=$ constant is precisely what we need to solve the cosmological constant problem, obtaining a cosmological term in agreement with observation [4]. It has also been shown [19] that the decaying cosmological term proposed by Chen and $\mathrm{Wu}$ is not the only feasible possibility, the equation of state $p=-\epsilon / 3$ being also compatible with a bicomponent content, formed by ordinary matter and a cosmological constant. Actually, this equation of state can correspond as well to textures or strings. However, as commented in Ref. [19], it would be unrealistic to consider that the present universe is dominated by such topological defects.

Finally, a curious remark is in order. With the superior limit for the matter angular velocity $\omega \sim 10^{-21} s^{-1}$, derived in section 2, the radius of the Universe $a \sim 10^{26} \mathrm{~m}$, and the matter density $\rho \sim 10^{-27} \mathrm{Kg} / \mathrm{m}^{3}$, we obtain an angular momentum of order $L \sim 10^{82} \mathrm{~J}$.s. With this value, it follows that

$$
\frac{L}{\hbar} \sim 10^{116} \sim\left(10^{39}\right)^{3},
$$

where $\hbar$ is Planck's constant. This relation between the angular momentum of the Universe and typical angular momenta of particles is also expected on the basis of the large number coincidences [20, 21]. Its physical meaning and cosmological implications remain an exciting and challenging open problem.

\section{ACKNOWLEDGMENTS}

I am greatly thankful to Guillermo A. Mena Marugán, without whose criticisms, suggestions and encouragement this work would have not been possible. I am also grateful to Luis J. Garay for his careful reading of a first version of the manuscript, to Mariano Moles for a useful discussion on galaxies formation, to 
Hugo Reis and Henrique Fleming for discussions on the Hubble law in anisotropic models, to Pedro F. González-Díaz for interesting discussions and warm hospitality in CSIC (Madrid), and to Enric Verdaguer and Alberto Saa for their hospitality in University of Barcelona during the Summer of 2000. My thanks also to Mariam Bouhmadi for her kind help with GRTensor and Mathematica. This work has been partially supported by CNPq.

\section{REFERENCES}

1. Korotkii, V. A., and Obukhov, Yu. N. (1991). Sov. Phys. JETP 72, 11.

2. Obukhov, Yu. N. (1992). Gen. Rel. Grav. 24, 121.

3. Gödel, K. (1949). Rev. Mod. Phys. 21, 447. Reprinted in Gen. Rel. Grav. 32 (2001) 1409.

4. Chen, W., and Wu, Y. S. (1990). Phys. Rev. D 41, 695.

5. Li, L. X. (1998). Gen. Rel. Grav. 30, 497.

6. Saibatalov, R. X. (1995). Gen. Rel. Grav. 27, 697.

7. Rebouças, M. J., and Tiomno, J. (1983). Phys. Rev. D 28, 1251.

8. Radu, E. (1998). Phys. Lett. A 247, 207.

9. Landau, L. D., and Lifshitz, E. M., The Classical Theory of Fields, Pergamon, Oxford, 1983, Secs. 111-114.

10. Weinberg, S. (1972). Gravitation and Cosmology, John Wiley \& Sons, New York, Sec. 15.6.

11. Hawking, S. W., and Ellis, G. F. R. (1973). The Large Scale Structure of Space-time, Cambridge University Press, Cambridge.

12. Perlmutter, S. et al. (1999). Astrophys. J. 517, 565.

13. Riess, A. G. et al., (1999). Astronomical J. 116, 1009.

14. Carneiro, S., and Mena Marugán, G. A. (2001). Phys. Rev. D 64, 083502.

15. Weinberg, S., op. cit., Sec. 2.11.

16. Misner, C. W., Thorne, K. S., and Wheeler, J. A. (1973). Gravitation, Freeman, New York, Sec. 22.3.

17. Carneiro, S., (2000). Phys. Rev. D 61, 083506.

18. Wesson, P., in R. Matthews, New Scientist, 19/26 December 1998 - 2 January 1999, p. 19.

19. John, M. V., and Joseph, K. B. (2000). Phys. Rev. D 61, 087304.

20. Carneiro, S. (1998). Found. Phys. Lett. 11, 95.

21. Dirac, P. A. M. (1937). Nature (London) 139, 323. 